\title{
Diagnosis of tuberculosis in patients with psoriasis: the need for a modified approach
}

\section{To the Editors:}

We read with interest the TBNET consensus statement on the diagnosis and treatment of tuberculosis (TB) in patients screened for tumour necrosis factor (TNF)- $\alpha$ antagonist therapy [1]. Admittedly, accumulated evidence during the last decade demonstrates that adherence to such guidelines has minimised the risk of TB reactivation in patients receiving immunosuppressive regimens, including anti-TNF- $\alpha$ therapy. For TNF- $\alpha$ antagonists specifically, targeted preventive chemotherapy prior to the onset of therapy has significantly reduced the risk of progression of latent TB infection (LTBI) to overt disease, yet at the cost of increased risk for significant treatment-associated toxicity. Thus, in an effort to avoid possible over-treatment prior to treatment with TNF- $\alpha$ antagonists and to accurately identify patients who would benefit from anti-TB treatment, patient cohorts inherently predisposed to overdiagnosis of LTBI should be identified. In the TBNET consensus statement, the author panel concludes: "Screening for latent infection and preventive chemotherapy against TB should not be different for patients with different underlying disease [...] who are candidates for TNF antagonist therapies (evidence level D)" [1]. However, in all these cases, the diagnosis of TB is performed in patients with overt, usually moderate-to-severe underlying disease to be treated with TNF- $\alpha$ antagonists.

Recent data have suggested that in a subpopulation of psoriasis patients, recommendations for TB diagnosis and management should be adapted to the organ (i.e. skin)-specific pathophysiological situation. Psoriasis is a disease characterised by inherently activated innate immunity long known to be connected to the presence of enhanced specific anti-TB immunity and a decreased risk of TB infection (discussed in [2]).

Applying tuberculin skin testing (TST)- $[2,3]$ or interferon- $\gamma$ release assay (IGRA)-based guidelines [4], recent studies have reported unexpectedly high rates of LTBI diagnosis among patients with psoriasis evaluated for anti-TNF- $\alpha$ treatment. Recent results from our hospital (University of Ioannina Medical School, Ioannina, Greece) have shown that TST outcomes are significantly higher in patients with plaque psoriasis compared with patients with other dermatologic diseases $(p=0.01)$ or diseases primarily affecting organ systems other than the skin $(p<0.0001)$ [2]. Moreover, in patients with psoriasis, the TSTs correlated with the burden of skin disease, as measured by psoriasis area and severity index $(p=0.015)$, indicating a direct pathophysiological relationship of skin reaction to tuberculin and clinical psoriasis activity [2]. Similarly, in a study from Switzerland, TST screening led to the putative diagnosis of LTBI in a comparably high proportion $(\sim 40 \%)$ of psoriasis patients evaluated for anti-TNF- $\alpha$ treatment [4].
However, even after applying an IGRA-based diagnostic approach, $20 \%$ of psoriasis patients screened for anti-TNF- $\alpha$ treatment were eventually treated for LTBI. This was still an unexpectedly high proportion for a country such as Switzerland, which has a very low incidence of overt TB [4]. These findings suggest an increased risk of false-positive LTBI diagnosis in patients with psoriasis, according to current official recommendations. According to a retrospective analysis from France, which applied the corresponding guidelines of the French Medicines Agency, two out of three psoriasis patients screened for anti-TNF- $\alpha$ initiation and diagnosed with LTBI were eventually over-treated for this latter condition. Notably, some degree of hepatotoxicity attributed to LTBI treatment developed in $40 \%$ of these patients [5]. These observations are further supported by recent studies by us and others indicating that, when evaluated according to officially recommended guidelines for TB prior to treatment with biologics, patients with moderate-to-severe plaque psoriasis or psoriatic arthritis are at a higher risk of being diagnosed with LTBI than patients sharing the same demographic background with inflammatory bowel diseases (Crohn's disease, ulcerative colitis) [3] or rheumatoid arthritis [6]. In addition, some patients with extensive psoriasis might not have healthy skin islands available for TST measurement; this sporadic event may be a decisive hurdle for the proper application of the Mantoux method.

The accumulated evidence discussed here leads us to conclude that current TB diagnosis recommendations, which are unbalanced for underlying disease, are likely to lead to LTBI overdiagnosis in patients with psoriasis and psoriatic arthritis, prior to anti-TNF- $\alpha$ treatment. In TST reaction in particular, it has been suggested that injection of tuberculin into the unaffected, yet "psoriasis-conditioned", pro-inflammatory skin of patients with overt plaque psoriasis triggers an augmented inflammatory response resulting in higher TST results due to an inherent overall "skin alertness" towards Mycobacterium tuberculosis antigens [2, 3]. We think that a re-assessment of the official LTBI diagnosis algorithms and their adaptation to the particular situation of psoriasis patients is needed. As recently proposed by GOUJON et al. [5], it should be prospectively evaluated whether an approach combining TST- and IGRA-based methods, like that currently recommended for paediatric patients [1], may be more suitable to the specific situation of TB diagnosis in patients with psoriasis. Finally, studies comparing TST and IGRA outcomes in patients with defined pathophysiological deviations should also contribute to better understanding of each of the above methods.

\section{I.D. Bassukas*, G. Gaitanis* and S.H. Constantopoulos ${ }^{\#}$}

Depts of *Skin and Venereal Diseases, and "Pneumonology, University of Ioannina Medical School, Ioannina, Greece. 
Correspondence: I.D. Bassukas, Dept of Skin and Venereal Diseases, University of Ioannina Medical School, 45110 Ioannina, Greece. E-mail: ibassuka@cc.uoi.gr

Statement of Interest: None declared.

\section{REFERENCES}

1 Solovic I, Sester M, Gomez-Reino JJ, et al. The risk of tuberculosis related to tumour necrosis factor antagonist therapies: a TBNET consensus statement. Eur Respir J 2010; 36: 1185-1206.

2 Tsiouri G, Gaitanis G, Kiorpelidou D, et al. Tuberculin skin test overestimates tuberculosis hypersensitivity in adult patients with psoriasis. Dermatology 2009; 219: 119-125.

3 Bassukas ID, Kosmidou M, Gaitanis G, et al. Patients with psoriasis are more likely to be treated for latent tuberculosis infection prior to biologics than patients with inflammatory bowel disease. Acta Derm Venereol 2011; [Epub ahead of print DOI: 10.2340/000155551106].

4 Laffitte E, Janssens JP, Roux-Lombard P, et al. Tuberculosis screening in patients with psoriasis before antitumour necrosis factor therapy: comparison of an interferon- $\gamma$ release assay versus tuberculin skin test. Br J Dermatol 2009; 161: 797-800.

5 Goujon C, Gormand F, Gunera-Saad N, et al. Pertinence des critères diagnostiques de tuberculose latente avant traitment du psoriasis par anti-TNF- $\alpha$. [The relevance of diagnostic criteria for latent tuberculosis before initiation of TNF-alpha inhibitors in psoriasis patients.] Ann Dermatol Venereol 2010; 137: 437-443.

6 Fuchs I, Avnon L, Freud T, et al. Repeated tuberculin skin testing following therapy with TNF- $\alpha$ inhibitors. Clin Rheumatol 2009; 28: 167-172.

DOI: $10.1183 / 09031936.00016611$

\section{From the authors:}

We thank our colleagues for their comment on the TBNET consensus statement on the risk of tuberculosis (TB) related to tumour necrosis factor (TNF) antagonist therapies [1]. The authors correctly point to a persistent diagnostic dilemma: the diagnosis of "true" latent infection with Mycobacterium tuberculosis [2] and the lack of knowledge on the positive predictive value for the development of TB offered by the two currently available immunodiagnostic methods, the tuberculin skin test (TST) and interferon- $\gamma$ release assays (IGRAs), in a variety of clinical circumstances [3].

By definition, the diagnosis of latent infection with $M$. tuberculosis relies on the presence of a positive M. tuberculosisspecific immune response in a TST or an IGRA. However, the immunological diagnosis of latent infection with $M$. tuberculosis is a relatively poor approximation of "true" latency [2]. The concept of preventive chemotherapy relies upon the identification of individuals who are at highest increased risk for the development of TB by positive $M$. tuberculosis-specific immune responses. Screening and treatment for latent infection with M. tuberculosis is only effective, efficacious and efficient when populations with a per se increased risk for the future development of TB are targeted [4]. These include recent close contacts of contagious index cases, individuals with HIV infection, subjects with silicosis, candidates for TNF antagonist therapies, patients with chronic renal failure, individuals with immunosuppressed stem cell, solid organ recipients, and others [5].

While the risk for the development of TB is strikingly different among patients, depending on the absence or presence of a specific risk factor [6], percentages of positive M. tuberculosisspecific immune responses are also heterogeneous when comparing groups of patients at increased risk for the development of TB. At the group level, population epidemiology matters. For example, in Europe, positive TST and/or IGRA responses have been observed in only $10-15 \%$ of individuals with HIV infection, compared with $\sim 25 \%$ of patients with chronic renal failure [7]. But the risk for the development of TB is higher among individuals with HIV infection than in patients with chronic renal failure [5]. What we are able to observe is the combined effect of underlying prevalence of infection, which we try to estimate more or less successfully with a test and the risk of TB given actual latent infection. Although the underlying mechanism may be different, the reported high percentage of positive $M$. tuberculosis-specific immune responses in patients with psoriasis [8] might not be indicative that psoriasis patients with a positive TST and/or IGRA response benefit as much from preventive chemotherapy against TB as other candidates for TNF antagonist therapies.

We agree with our colleagues that the percentage of positive M. tuberculosis-specific immune responses is likely to vary between different groups of patients with candidates for TNF antagonist therapies. The probability of a positive TST or IGRA is, among other factors, certainly related to the underlying clinical condition. As the predictive value of a positive test hinges largely on the prevalence and individual future morbidity risk, as well as on the absence or presence of defined risk factors and their magnitude if present, it would therefore be critical to obtain more precise information separately for each group of individuals at potentially increased risk of TB. However, until we have such evidence, we should be cautious and state, using the lowest possible evidence grading of " $\mathrm{D}$ ", that screening for latent $M$. tuberculosis infection and preventive chemotherapy against TB should not be different for distinctive groups of patients with underlying diseases (rheumatoid arthritis, psoriasis, inflammatory bowl disease) who are candidates for TNF antagonist therapies.

\section{Lange*, H. Rieder", M. Sester ${ }^{*}$, H. Milburn ${ }^{+}$, B. Kampmann ${ }^{\S}$, G.B. Migliori ${ }^{f}$ and I. Solovic**}

*Research Center Borstel, Borstel, and 'Saarland University, Homburg, Germany. "International Union against Tuberculosis and Lung Disease, Paris, France. ${ }^{+}$Guy's and St Thomas Hospital, and ${ }^{\S}$ Imperial College, London, UK. ${ }^{f}$ Fondazione S. Maugeri, Tradate, Italy. ${ }^{* *}$ Catholic University, Ružomberok, Slovakia.

Correspondence: C. Lange, Division of Clinical Infectious Diseases, Tuberculosis Center Borstel, Medical Clinic, Parkallee 35, 23845 Borstel, Germany. E-mail: clange@ fz-borstel.de

Statement of Interest: None declared. 